Itinéraires Itinéraires

Littérature, textes, cultures

2018-1| 2018

Le manifeste à travers les arts : devenirs d'un genre indiscipliné

\title{
Manifestes en performance : de quelques manifestes de poésie expérimentale
}

Manifestos in Performance: On Some Manifestos of Experimental Poetry

\section{Gaëlle Théval}

\section{OpenEdition}

\section{Journals}

Édition électronique

URL : http://journals.openedition.org/itineraires/4399

DOI : $10.4000 /$ itineraires.4399

ISSN : 2427-920X

Éditeur

Pléiade

Référence électronique

Gaëlle Théval, « Manifestes en performance : de quelques manifestes de poésie expérimentale », Itinéraires [En ligne], 2018-1 | 2018, mis en ligne le 15 septembre 2018, consulté le 16 mai 2020. URL : http://journals.openedition.org/itineraires/4399; DOI : https://doi.org/10.4000/itineraires.4399

Ce document a été généré automatiquement le 16 mai 2020.

\section{cc) $(9)$}

Itinéraires est mis à disposition selon les termes de la licence Creative Commons Attribution - Pas d'Utilisation Commerciale - Pas de Modification 4.0 International. 


\title{
Manifestes en performance : de quelques manifestes de poésie expérimentale
}

\author{
Manifestos in Performance: On Some Manifestos of Experimental Poetry
}

\author{
Gaëlle Théval
}

1 Concrète, visuelle, sonore, phonique, phonétique, action, directe, élémentaire, spatialiste, séméiotique, objective, la multiplicité des épithètes utilisées pour décrire des pratiques naissant au cours de la décennie 1960-1970 crée un effet paradoxal : si le retour des mêmes noms, les croisements au sein de revues, de festivals, donnent malgré tout l'impression d'une forme d'unité, d'air de famille entre ces pratiques, les épithètes multiples contribuent à donner du champ une image hétérogène, fragmentée, difficile à appréhender et identifier comme avant-garde, à l'instar de ce que fut Tel Quel. Pourtant, les évolutions poétiques récentes, les nombreuses pratiques performatives et intermédia et le brouillage toujours plus marqué du champ de la poésie contemporaine, conduisent à une réévaluation de ce qui apparaît désormais comme un héritage incontournable. Or, outre la problématique du canon littéraire, l'examen de cette période et en particulier des pratiques manifestaires qui y ont cours, permet de rendre sensible une évolution quelque peu différente de ce que l'histoire littéraire retient bien souvent comme le moment de la « mort des avant-gardes".

2 Notre étude se focalise sur trois manifestes, qui, sans être les seuls abordés, apparaîtront comme des balises pour arpenter le champ et l'histoire des poésies expérimentales en France, produits entre 1961 et 1978 par trois figures centrales du domaine : Pierre Garnier, Bernard Heidsieck et Julien Blaine. Fondateurs ou directeurs de revues, Pierre Garnier ${ }^{1}$ et Julien Blaine ${ }^{2}$ ont joué un rôle majeur dans la production et la diffusion de ces pratiques. Si Bernard Heidsieck ne dirige pas de revue il est en lien étroit avec Henri Chopin, fondateur de la revue OU/Cinquième saison, revue-disque principal support de diffusion de la poésie sonore, et a contribué par d'autres actions (organisation de festivals, notamment) à la diffusion de la poésie sonore. 
Ces manifestes ont pour premier point commun d'en revendiquer le statut par leur titre, ou leur désignation postérieure, et par certains de leurs traits caractéristiques. Ils font partie de ces productions qui sont "plus clairement des manifestes que d'autres" (Tomiche $2005: 6$ ), sans apparaître pour autant toujours sous une forme textuelle. Les textes publiés par Pierre Garnier dans la revue Les Lettres entre 1962 et $1964^{3}$ se désignent ainsi clairement, comme le «Manifeste pour une poésie nouvelle, visuelle et phonique » (daté du 30 septembre 1962), et le "Deuxième manifeste pour une poésie visuelle " (daté du 31 décembre 1962). Les deux suivants, «Position 1 du mouvement international » et «Position 2 du spatialisme », respectivement datés du 5 août 1963 et du 10 septembre 1964, se présentent à leur tour comme des manifestes « stricto-sensu " (Abastado 1980: 4), en ce sens qu'ils revendiquent une prise de parole au nom d'un mouvement qu'ils prétendent fonder: le "Spatialisme». Antérieur d'une année, le texte sans titre de Bernard Heidsieck, que l'on désigne par ses premières lignes « Pour un poème debout... » édité par la Galerie internationale d'art contemporain à l'occasion de l'exposition 7 Métasignes de Jean Degottex, est appelé par ce dernier "page manifeste " (Heidsieck 2001: 194) puis «texte/manifeste» et diffusé dans Cinquième saison en $1962^{4}$ :

Je me suis depuis toujours réjoui qu'à travers ce TEXTE/MANIFESTE et l'évocation de ce Poème-Partition $\mathrm{D} 3 \mathrm{Z}$ qui lui était dédié, se soient trouvés associés son nom et l'apparition pour la première fois de ma part de la notion de POÉSIE DEBOUT avec sa revendication nettement et hautement affirmée. (Heidsieck $2008: 9$ )

Si Couper n'est pas jouer fait entendre la rumeur de la manifestation de mai 68, c'est de "préface» que le poète qualifie cette pièce sonore ${ }^{5}$. Cependant, celle-ci remplit la fonction de manifeste, ou d' "archi-préface", non seulement parce que, comme le rappelle Jean-Marie Gleize sur le plan fonctionnel «tout manifeste est une préface en ce sens qu'il sert de préface générale à un ensemble d'œuvres possibles » $(1980: 12)$, mais aussi dans la mesure où cette "préface - triface " se situe dans cette position au regard de l'œuvre tout entière et non d'un ouvrage qu'elle viendrait présenter ${ }^{6}$. Enfin parmi les nombreux manifestes produits par Julien Blaine, c'est à deux textes et une performance que nous nous intéresserons : «La poésie hors du livre hors du spectacle hors de l'objet ", cosigné avec Alain Schifres et Jean-Claude Moineau, manifeste paru dans le numéro 5/6 de Robho en 1971, la performance "Quant au livre de l'échec/Quant à l'échec du livre", datée de 1972, qualifiée par le poète de «manifeste» (Blaine 2009: 87) et le "Manifeste pour l'occupation des socles et des stèles abandonnés ", publié sous forme d'affiche et envoyé au réseau mail art en 1978.

Comme stratégie discursive, le manifeste est geste avant-gardiste par excellence, lié à la fondation d'un mouvement, à l'inscription d'un collectif (Margel 2013: 5). Les années 1960-1970 représentent un moment de transition, entre redécouverte des avant-gardes historiques et expérimentations nouvelles, laissant advenir les "néoavant-gardes ", auxquelles Peter Bürger ([1974] $2013: 86)$ reproche de rejouer les gestes avant-gardistes pour en falsifier le projet transgressif. Le manifeste revêt cependant des formes qui, dans ce contexte, en pluralisent les modes d'action.

C'est donc tant aux contenus critiques et programmatiques qu'aux modalités d'action de ces manifestes que nous nous intéresserons, s'il est entendu que le manifeste ne saurait se réduire à un genre mais, est « un geste, un acte ", relevant " prioritairement d'une pragmatique du discours, d'une lecture sociologique, d'une analyse en termes de stratégies d'intervention dans le champ de l'institution» (Gleize 1980:12), parce qu'intervenant « dans une situation historique qu'il a à dessein de faire signifier et de 
transformer à la fois » (Vouilloux $2004: 126)$. Qu'en est-il de l'acte manifestaire dans la poésie expérimentale et en particulier dans la "poésie action"? Notre propos s'articulera autour de trois actions dont il s'agira d'observer les modalités : sortir du livre, où les programmes énoncés nous arrêterons, faire exister un groupe, où les modes de circulation ainsi que les impacts de ces manifestes seront envisagés, et agir la poésie, où la spécificité du mode d'agir des manifestes de poésie action sera interrogée.

\section{Sortir du livre}

7 Reçu comme texte de rupture et de fondation, le manifeste a une propension didactique et «fonctionne comme un mythe»: «Dans un remodelage manichéen de la temporalité, le passé est décrit comme de la non-vie, ou comme le temps de gestation de la vraie vie» (Abastado 1980: 6). Les trois ensembles manifestaires examinés fonctionnent sur ce mode. Il s'agit pour ces poètes de s'inscrire en rupture, moins pour revendiquer une esthétique nouvelle qu'une véritable révolution médiologique. La convergence des programmes y est cependant partielle, notamment quant à leur visée politique.

\section{« La poésie piétine »... en plaquettes}

8 Le commun constat est celui d'un état moribond du champ poétique durant les années 1950. Derniers feux du surréalisme, poésie «engagée » ou «blanche », d'une part, avant-gardisme textualiste et théoricien de Tel Quel d'autre part, sont renvoyés dos à dos dans un sentiment de lassitude partagé : « Nous piétinons. L'esprit tourne. La poésie piétine", annonce Pierre Garnier dans son "Manifeste pour une poésie nouvelle, visuelle et phonique ». Or cet ennui est relié, dans les discours, à un repli de la poésie sur elle-même, dans une attitude autoréflexive confinant au nombrilisme, dont la traduction médiologique est un certain fétichisme de l'objet livre. Si la critique de Garnier reste discrète, la "plaquette » se voit affublée d'une connotation surannée : "Avec lassitude nous prenons connaissance de nos propres plaquettes" (Garnier [1963a] $1968: 129)^{7}$. Les évolutions technologiques, liées au développement des médias de masse et à la démocratisation d'appareils enregistreurs comme le magnétophone, lui font affirmer plus loin: "Le temps des livres semble passé. On a voulu graver sur disques la poésie ancienne ; le résultat fut presque toujours désastreux, parce que cette poésie a été écrite pour le livre » (Garnier [1963a] 1968 : 135).

9 Le rejet du livre comme lieu d'un repli du poème sur lui-même est au centre des deux manifestes de Bernard Heidsieck. Le texte de "pour un poème debout... » adopte les caractéristiques discursives de l'écrit manifestaire de manière systématique, en forçant presque les traits. Exhibant une structure tripartite, il débute par un " pour ", prise de parti pour un " poème debout ", auquel s'oppose plus loin un « contre » dirigé vers une "poésie point de mire ", autocentrée, qui se complaît dans le médium livresque au point d'y risquer la noyade. Une poésie qui n'est capable d'offrir au lecteur, « au terme du cycle et de sa trajectoire, que le reflet blanc d'une glace sans tain ou le trou noir d'une poésie cul-de-sac» (Heidsieck 2001 : 165). La page sera alors réduite au rôle de «tremplin » pour la "quête d'oxygène » d'un poème « debout ». "La poésie écrite n'a plus lieu d'être ", lit-on dans le texte de Couper n'est pas jouer, composé d'extraits de 
" Notes convergentes. Poésie action et magnétophone ", alors inédit ${ }^{8}$, et le poème doit se résoudre "à ne plus être l'objet seul de ses caresses et de ses délectations".

Chez Julien Blaine, le livre se voit également mis en cause comme lieu où la poésie ne peut qu'étouffer, en raison de la charge symbolique du livre comme espace clos, d'une part, comme objet marchand d'autre part. La performance « Quant au livre de l'échec / Quant à l'échec du livre » établit un dialogue à distance avec le projet mallarméen, pour se donner comme " un manifeste contre le livre tel qu'il était considéré à l'époque : unique support de l'écrit, inerte et intouchable, posé sur son piédestal et affublé d'une charge symbolique qui n'aide pas la diffusion des idées, bien au contraire » (Blaine 2009 : 87). La performance donne à voir le poète, jouant aux échecs sur « un damier géant », à l'aide de pièces constituées de fragments d'imprimés. Lorsque la partie arrive à confusion, le poète prend le damier, fait «corps avec lui comme dans une lutte, en déchire les cases, les mêle aux pièces, les relie entre elles pour fabriquer un nouveau livre. Ce dernier est voué à être lu, mais également à être détruit » (Blaine 2009: 89). Le livre est également fustigé en tant qu'objet, que la « poésie deux points » revendiquée dans le manifeste de 1971 rejette au même titre que le spectacle, comme symptôme d'une société mercantile. Là encore, la rhétorique manifestaire, assortie d'une tonalité pamphlétaire, joue à plein, le texte commençant par aligner "ses refus" avant d'exposer son programme. Tendue vers l'action cette poésie s'«érige contre la séduction du livre, et plus généralement, de l'objet dont l'objet-livre (Gallimard, Le Seuil, Maeght), au même titre que le livre-objet (Le Soleil Noir, Givaudan) n'est qu'une variante mystifiée, une marchandise canonisée » (Blaine 2009 : 89).

\section{« L'arracher à la page... »}

11 À ce constat sera alors opposée la nécessité de sortir le poème du livre, pour utiliser les technologies nouvelles à disposition comme moyens de diffusion mais aussi de création.

La rhétorique injonctive du manifeste est à l'œuvre dans les appels de Pierre Garnier qui, s'adressant au lecteur / créateur, affirme : « Les mots doivent être vus. [...] Libérez les mots. Respectez les mots. Ne les rendez pas esclaves des phrases. Laissez-les prendre leur espace. [...] Il faut pulvériser notre langage usé - c'est-à-dire faire scintiller le mot ", dans une proposition qui n'est pas sans rappeler celle du Marinetti des Mots en liberté. Libéré de la phrase, le mot est alors non plus pris dans sa forme mais « donné dans sa force» («Position $2 \mathrm{du}$ spatialisme »). Tendue vers une objectivation du langage, la poésie revendiquée par Garnier se donne pour visée de créer des poèmes qui soient des objets « poèmes-objets qui existeront à un seul exemplaire » (1963b : 16), de façon à opérer la distanciation nécessaire que le livre ne permet pas. Le poème se fera œuvre unique, et s'inscrira sur des supports autres pour « rejoindre l'univers » et «se perdre dans les espaces $»$ :

Il n'est pas question de cantonner la poésie visuelle sur le papier qui, par sa banalité, sa platitude, son neutralisme, est un mauvais porteur. Il faudra inscrire le poème sur des murs, sur des pierres, sur des vitres, sur du sable figé, sur du papier d'emballage, sur de vieux sacs. (Garnier 1963b : 18)

13 Le poème devient alors «quelque chose de mesurable, de préhensible, non seulement pour la vue, mais pour l'ouïe, pour l'odorat, pour la main » (1963b : 18). Par la suite, le caractère relativement statique de l'objet est supplanté par une vision de la « languematière » comme "énergie active ». Partant, la page n'est plus nécessairement le 
support privilégié du poème spatial, et le poème est rendu indissociable de son médium :

Or le moyen technique employé crée la poésie autant que le poète. Le magnétophone, le disque, la télévision doivent créer leur propre forme de poésie. [...] L'emploi du magnétophone transforme radicalement la forme de la poésie, donc son contenu. Il est possible d'« écrire » sur la bande magnétique comme on écrit sur la feuille blanche. (Garnier [1963a] 1968 : 135)

Après une première phase de production de « poèmes partitions » entre 1955 et 1958, destinés à la lecture, l'acquisition d'un magnétophone en 1959 entraîne une première inflexion, Heidsieck se mettant à enregistrer les poèmes en question, et à en envisager la publication sur disque. Puis, à partir de 1961, et du poème-partition «D3Z », il commence à utiliser le magnétophone non plus comme simple moyen d'enregistrement de sa voix, mais aussi comme moyen de captation de sons extérieurs, et bientôt, comme moyen d'écriture à part entière, intervenant directement sur la bande pour effectuer un travail de montage. Cette date marque aussi la première diffusion publique d'un poème-partition, qui coïncide avec l'écriture et la diffusion du premier manifeste, «Pour un poème donc... ». Filant la métaphore du poème «debout », «dressé », « les pieds sur la page ", le poète sonore oppose la poésie action à une poésie du livre " couchée ", "dans l'attente du client ", marquée par la passivité. "L'arracher à la page » : les métaphores décrivent un geste plus violent dans Couper n'est pas jouer : « La poésie-action est née, naît, dès le premier soupçon d'arrachement à la page : à cet instant exact de fébrilité où tout s'est ébranlé et rompu, où tout s'ébranle et se rompt " (Heidsieck [1968] 2005 : 22). Et si le poème est arraché à la page, c'est pour se voir " projeté » dans l'espace, celui de la performance :

Et que s'ouvrent, s'écarquillent les yeux, ne serait-ce que l'espace seul de sa durée, et se braquent

et se branchent

sensibilisés

sur un geste,

puis deux...

l'ouie, la peau, les nerfs, électrisés.

Le vocabulaire décrit, comme chez Garnier, une forme de tension, mettant en jeu et en action le corps dans son entier, celui-là même que la poésie performance de Blaine fait intervenir dans sa lutte au corps à corps avec l'objet livre. Le mouvement d'arrachement se double d'une projection: la poésie sera action, parce qu'agie par le poète, mais aussi par sa capacité à circuler et agir dans la société.

\section{Circulations : poésie et société}

La sortie du livre s'accompagne en effet, chez Heidsieck, de l'énoncé d'une volonté de remise en circulation de la poésie dans la société. L'association de métaphores guerrières et picturales décrit le mode d'action voulu pour le poème : "projeté aussi, mobile, errant, à l'affût / à l'assaut des corps ", assaut implicitement comparé à la morsure de l'eau-forte, faisant du poème non pas un objet, mais un acide : «pour une action immédiate et physique du poème, avec brûlures d'acide sur plaques de cuivre ou de marbre et de chair, le désir, aussi, surtout, de s'y blottir, glisser, coller, plaquer.» Dans Couper n'est pas jouer, le propos s'attarde sur le constat d'une mutation sociétale laissant apparaître un nouvel ordre : 
[...] tandis que suintent, ici, là, un peu partout, se profilent, s'installent et prennent racine les prémices et mille signes et indices d'un nouvel ordre coercitif d'autant plus efficace, redoutable [...] ... que se mettent en place et s'édifient des structures avec notre aide et notre appui quotidiens - malgré nous - que se métamorphose le monde, de fond en comble [...] ... et que les cellules, noyaux, rivets, cadres, grilles, artères, moteurs, camisoles, tubes de cette charpente en cours d'édification se nomment: Production. Production? Productivité. Planification - souple ou nonProduction, Publicité, Presse (ah! le cœur), Polices (très proliférantes et de toutes sortes) ... Pouvoirs Publics (très prolixes), Pouvoir Pensant (certes, mais par procuration seulement), et bien d'autres « $\mathrm{P}$ » encore, d'autres Pressions, pouah! jusqu'aux Plaisirs (loisirs est plus correct) populaires poliment placés sous plombage... (Heidsieck [1968] $2005: 9$ )

17 Et le poème, alors, de s'ouvrir à ces «bruits » : «... Le poème se retourne de $180^{\circ}$ et s'ouvre au monde. Il est à réinventer. » L'on retrouve, dans ce second manifeste, la formule d'adhésion déployée dans le premier, assortie de métaphores ménagères, désignant le poème non plus comme objet clos, bibelot, mais comme moyen, tendu vers l'action la plus prosaïque: "Pour un poème-éponge... pour un poème serpillère » (Heidsieck [1968] 2005 : 24). "Rendu au sol », le poème se voit dépouillé de sa vocation esthétique pour être investi d'une visée pratique. En ce sens, le projet est éminemment politique : c'est l'autre sens de la "poésie action », qui se positionne au cœur de la société et non dans ses marges ou dans un ailleurs. Le manifeste définit et propose des modes d'agir :

L'État, la Puissance publique, son appareil et ses pompes, à partir de ces données collectives, se fixent des objectifs globaux, pensent, légifèrent et réglementent par grandes masses et agissent sur ce capital humain [...]. Le poème, en se glissant, en se situant à la charnière de ces forces contradictoires, au point précis de leur conjonction, [...] ou trancher aussi, éventuellement dans cette chair de signes, de codes, d'interdits, d'impératifs et de signalisation. Tout ceci au cœur même de la forteresse, en agissant de "l'intérieur ", plutôt que d'inlassablement crier, criailler, geindre et pleurer à sa porte, les yeux braqués sur un âge révolu. (Heidsieck [1968] $2005: 36)$

Situé au cœur du bruit, le poème peut prétendre y agir, rejoignant de la sorte le projet avant-gardiste, tel que le définit Peter Bürger, tourné non pas «contre une manifestation antérieure de l'art (un style), mais contre l'institution art en tant qu'institution séparée de la vie pratique des hommes » (Bürger $2013: 82$ ).

atialisme de Pierre Garnier doit aussi, dans les intentions qu'il affiche, conduire la poésie à regagner l'espace quotidien, mais selon une logique aux visées moins immédiatement politiques. Le poème vise à rejoindre le quotidien. Pour Garnier en effet, l'homme n'est, aujourd'hui, « plus déterminé par son milieu, par son peuple, par sa classe, mais par les images qu'il reçoit, par les objets qui l'entourent, par l'univers » (Garnier 1963b : 20). Dès lors, pour agir, la poésie devra sortir du livre pour « entrer dans les villes et les demeures » en se fondant aux objets domestiques - selon une stratégie qui n'est d'ailleurs pas sans rappeler celle de la publicité à la même époque : "l'affiche, les poèmes visuels en céramique, les calendriers faits de poèmes visuels, [...] des poèmes visuels mobiles en néon, des poèmes visuels sur cartes postales, des boîtes où seront enfermés quelques mots, des tapisseries - enfin, et peut-être surtout, le cinéma " (Ibid.). Plutôt que de prétendre délivrer "sentiments ou connaissance », le poète fera en sorte que la poésie « nous tienne compagnie » (Ibid.).

C'est précisément contre cet art du bibelot décoratif que représente à ses yeux la poésie visuelle et spatialiste («ceux qui copient Marinetti et Apollinaire sur octavo-jésus ») 
que Julien Blaine prendra position. Le manifeste «La poésie hors du livre hors du spectacle hors de l'objet » déclare ainsi pour « champ d'action : une société donnée à un moment donné », pour «but : agir sur et dans la vie quotidienne » par « l'anonymat, le parasitage, la subversion » (Blaine 2009 : 52), et donne pour champ d'action à la « poésie deux points» le champ social: "Elle est un instrument dialectique de la vie quotidienne » :

L'écriture va DU MONDE AU MOT.

La poésie Deux points, elle, fait le chemin inverse : DU MOT AU MONDE.

Si le monde est un texte, encore faut-il y aller voir.

LES POÈTES DEUX POINTS, EUX, PRENNENT LE MONDE À LA LETTRE ET

CHOISISSENT DE LA MODIFIER. (Blaine 2009 : 52)

Le texte "Plate-forme de base pour les éveilleurs de jour et de nuit de la poésie ":" " précise que ceux qui se nomment désormais "éveilleurs" s'engagent à abandonner non seulement le livre mais aussi toute production d'objet, et de " consacrer l'essentiel de l'activité, jadis consacrée aux livres, aux objets, aux spectacles, aux expositions et aux revues, à parasiter le paysage urbain - à commenter matériellement les paysages ruraux et maritimes» (Blaine 2009: 60). La poésie d'action appelée par ces manifestes et ceux qui suivent, publiés dans les journaux de freepress comme Geranonymo à partir de 1972, dont Blaine est le directeur de publication, puis dans la rubrique de Libération consacrée au VAN (Vrai Art Nouveau), est une poésie qui se réalise dans l'action quotidienne, dans une optique de fusion de l'art et de la vie, et une volonté de greffe de l'acte « à même la réalité ».

\section{Faire mouvement ?}

Fruit le plus souvent d'une énonciation plurielle, le manifeste est, dans son histoire, un " acte fondateur d'un sujet collectif ». Selon Claude Abastado, le manifeste fonctionne comme une machine de désir, visant à « faire exister comme entité reconnue un groupe [...] animé par des convictions communes et le désir d'action » et a toujours " pour effet de structurer et d'affirmer une identité » (Abastado 1980: 7). Les années 1960 sont marquées de ce point de vue par une évolution dans les modes de structuration des avant-gardes. Le domaine de la poésie expérimentale est précisément le théâtre de stratégies divergentes, que la confrontation de nos trois ensembles manifestaires fait nettement apparaître.

\section{La tentative spatialiste}

La parution successive dans la revue Les Lettres de quatre manifestes sur une période de deux années, ainsi que l'évolution des sommaires et de la ligne éditoriale de la revue dont Pierre Garnier devient directeur à partir du numéro 32 montrent une tentative de mise en place d'un mouvement : le spatialisme.

Le « Manifeste pour une poésie nouvelle visuelle et phonique » parait dans un numéro entièrement dédié au poète. Dans ce numéro, le poète semble ainsi parler en son nom, exposant des recherches personnelles, et ouvrant, par le biais de la revue, à la mise en relation avec d'autres : cherchant non pas à signer l'acte de naissance d'un mouvement, mais bien à le susciter. Le manifeste esquisse des rapprochements, avec les travaux 
menés par Henri Chopin ${ }^{9}$ au même moment au sein de la revue Cinquième saison, autour de la " poésie objective» :

Henri Chopin et la Revue Cinquième Saison lancent, à la suite de longues recherches, la poésie phonétique et la poésie objective. Partant du même refus, mais à la suite d'autres essais et d'autres recherches, je propose la poésie visuelle et la poésie phonique. (Garnier [1963a] 1968 : 129)

Parallèlement, Henri Chopin publie un poème de Garnier dans le numéro 11 de Cinquième Saison, en 1961, puis, dans le numéro 18 au printemps 1963, un «Manifeste bref pour une poésie visuelle ", en note duquel Chopin renvoie au " Manifeste pour une poésie visuelle et phonique ». Dans les numéros suivants, Garnier opère un travail de compilation de manifestes émanant d'autres mouvements plus structurés (notamment ceux de la poésie concrète allemande et brésilienne), et de textes théoriques. Dès le numéro 30, la revue se dote d'un sous-titre, "Poésie nouvelle », et une " Note sur la poésie nouvelle " précise en ouverture :

Un peu partout dans le monde des essais équivalents sont faits qui seront les fondements d'un renouvellement de la poésie. [...] Partout des hommes sont à l'œuvre. Presque toujours solitaires, ils nomment diversement leurs tentatives: poésies concrètes, phonétiques, objectives, visuelles, phoniques, etc. Ils finiront par se rejoindre car tous ces essais n'ont qu'un but : sortir la poésie du langage et en faire un élément. (Garnier 1963b : 1)

Citant à nouveau les collaborations avec des peintres et musiciens dans les tentatives de poésie «objective » de Henri Chopin, le manifeste dote le mouvement en cours de constitution d'une généalogie ( Ces essais ont des précurseurs: on peut citer Morgenstern, Albert-Birot, Kurt Schwitters, Hugo Ball, Michel Seuphor, certains expressionnistes, certains surréalistes, certains lettristes.»), et donne une bibliographie. Le « Deuxième manifeste pour une poésie visuelle » témoigne encore de recherches en cours. Le numéro accueille un texte théorique du poète concret allemand Franz Mon, "Articulations", ainsi qu'un texte projectif d'Henri Chopin, "Les mutations poétiques", aux accents manifestaires, conjuguant un véritable geste au passé simple, à un usage prophétique du futur annonçant la poésie à venir: «En conclusion, la poésie objective est l'objectivation de nos langages dans l'inconnu que nous rendons visuel, et visible, la poésie phonétique est le chant de la vision " (Chopin $1963: 13)$.

La notion de "spatialisme » apparaît au numéro suivant, qui témoigne d'une étape supplémentaire dans la structuration du mouvement. Une « Note liminaire - Plan pilote fondant le Spatialisme » est présentée comme un projet à amender par ses potentiels signataires, et annonce la création d'un "Comité International qui devra publier un bulletin de liaison, organiser des manifestations, des expositions, des conférences " (Garnier 1963c: 1). Le manifeste se présente donc comme une proposition pour fusionner en un mouvement commun les tendances diverses que représentent la poésie concrète, phonétique, objective, visuelle, phonique, cybernétique comme autant de « recherches dans le sens d'une poésie qu'on peut couvrir du nom général de Spatiale (les notions de temps, de structure, d'énergie y étant incluses)» (Garnier 1963c:2). Et le poète de préciser par la suite leurs points communs (objectivation de la langue, destruction de l'idée d'œuvre au profit de celle d'« énergie transmise », dépassement des langues nationales). Le plan-pilote paraît à nouveau, assorti de quelques modifications et surtout co-signé ${ }^{10}$, dans le numéro 32, sous le titre «Position I du mouvement international », doté d'une énonciation explicitement collective, actant en 
apparence la naissance du mouvement «Spatialisme ». Le sous-titre de la revue change à nouveau, devenant "Poésie nouvelle-revue du Spatialisme», qui désigne la publication comme l'organe de diffusion du mouvement.

Cependant, au sein du même numéro, les premiers grincements se font entendre, qui donneront le la pour la suite: essentiellement paraphé par des poètes visuels et concrets, le manifeste n'est en effet pas signé par celui qui était pourtant présenté comme le premier compagnon de route, Henri Chopin. Ce dernier se fend en effet d'une réponse dans laquelle il exprime son soutien, mais aussi son refus de s'affilier au groupe, avançant moins dans ce texte une divergence poétique pourtant réelle, que le refus du modèle même du " groupe » et du " mouvement » :

Le mouvement dont nous sommes tous des «membres » a priori n'existe pas. Je ne suis pas membre d'un mouvement mais je suis «avec» le mouvement. Je suis le mouvement. [...] qu'ai-je à faire d'un mouvement, fût-il international! Cela, c'est bon pour un chef d'État qui parle de rayonnement universel d'un pays... C'est bon pour tous les songe-creux qui vulgarisent un "arrêt " qu'un jour un poète a proféré. (Chopin [1963b] 1968 : 142-143)

Le modèle avant-gardiste est mis à distance, voire franchement récusé par Chopin mais aussi par nombre d'autres poètes du paysage poétique expérimental. Julien Blaine refuse lui aussi l'idée de groupe, refus qui le tient également à distance des lettristes, puis des situationnistes, avec lesquels il partagera pourtant des conceptions similaires, et même de Fluxus : «j'étais en rejet total du Lettrisme, qui se construisait en décalque parfait des vieux mouvements comme le surréalisme, avec un maître, des lieutenants, des luttes de pouvoir, internes, stériles...» (Blaine 2009 : 47). La prudence est d'ailleurs de mise dans le volume Spatialisme et poésie concrète paru en 1968, où, les notices biobibliographiques d'auteurs "prenant part aux recherches, aux expériences, créant la poésie nouvelle", sont assorties d'une mise en garde: "Ces renseignements sont fragmentaires et donnés à titre indicatif. Ils ne lient pas leurs auteurs " (Garnier 1968 : 190). On y retrouve, pour la France, les noms de Julien Blaine, Jean-François Bory, Pierre Garnier, Bernard Heidsieck, Henri Chopin, et François Dufrêne. Pierre Garnier reconnaît l'échec de la tentative dans un entretien postérieur :

C'était une proposition pour rassembler une réalité en un mouvement ; j'avais peutêtre une idée assez fausse du mouvement à l'époque, parce qu'il y avait eu Dada, il y avait eu le surréalisme, il y avait eu l'expressionnisme, le futurisme, et il n'y avait pas de raison qu'il n'y ait pas de mouvement semblable dans les années 60. Mais il se peut que déjà l'époque des mouvements soit passée, et qu'à ce niveau-là j'aie été en retard, en ce qui concerne du moins la dénomination. (Donguy 1998 : 395)

\section{« La poésie sonore, ce n'est pas une école, mais c'est plus qu'un mouvement »}

Les manifestes publiés par Bernard Heidsieck ne prétendent quant à eux pas fonder un mouvement, modèle que le poète sonore récuse : dans un texte consacré aux avantgardes, il explique en partager l'«esprit de recherche et sa volonté novatrice» (Heidsieck 2001 : 181) mais en refuser l'esprit de groupe, dont le surréalisme et le plus récent lettrisme constituent les contre-modèles :

Il n'en reste pas moins que la structure du Groupe et son comportement d'ensemble, créé et vécu à l'image désuète - et comme sa répétition recherchée - de feu le Surréalisme - Pape et totalitarisme compris - revendications abusives, 
répétées, gratuites et risibles, et chapelet d'injures outrancières, ont eu de quoi désarmer les meilleures volontés. (Heidsieck 2001 : 191) modalités de création, puis de diffusion des manifestes. "Pour un poème debout..." " introduit en effet, à l'origine, le poème " $\mathrm{D} 3 \mathrm{Z}$ », consacré aux peintures de Jean Degottex, dont il se veut la transposition sonore ${ }^{11}$. La coprésence de ces deux textes aux statuts énonciatifs différents, l'un manifestaire, l'autre poétique, confère au second un statut particulier, exemplaire de ce que peut être cette "poésie debout " que décrit le premier, en même temps qu'il confirme une relation de spécularité entre la peinture et la poésie. C'est en passant par la peinture que le poète fait retour sur sa propre pratique poétique, pour en définir les orientations. En ce sens Heidsieck ne déroge pas à une certaine tradition avant-gardiste, et pointe une convergence des sensibilités. La circulation même du manifeste fait sens : il est d'abord distribué sur une feuille de grand format, durant l'écoute du poème « D3Z », lui-même diffusé lors du vernissage de l'exposition de Jean Degottex. L'intention est explicitée dans une lettre à Jean Fournier, alors directeur de la galerie Kleber où Degottex expose, qui accompagne l'envoi du premier poème consacré au peintre, « $\mathrm{D} 2$ » :

La «sensibilité » actuelle exige une forme identique de tension quelle que soit la forme d'art ou poétique choisie. En ce sens se répondent les silences ou «temps morts " - leur importance s'en décuplant - de la musique ou d'un poème et les surfaces unies de la peinture où vient se déployer le signe; le dynamisme éclaboussant de ce dernier et l'éclatement d'une gerbe de sons ou du verbe luimême ; le rythme identique avec lequel est exprimé le «signe ", enfin, né d'un ou plusieurs gestes, joué tel morceau ou dit ce poème (écueil peut-être ou naissance au contraire d'une poésie plus audible que lisible), ce rythme d'exécution donnant en quelque sorte l'apparence, non pas tant de s'écouler ou de se dérouler dans le temps, que d'attaquer l'instant [...]. Quoi qu'il en soit la notion d'agressivité pourra être considérée comme l'un des tout premiers dénominateurs communs possibles. (Heidsieck 2009 : 271-272)

L'écoute de «D3Z », comme ensemble de gestes sonores et miroir de la peinture de Degottex, oriente alors vers la recherche d'une analogie entre la poésie sonore, telle qu'elle s'élabore au cours des années 1950, et la peinture gestuelle, qui semble pouvoir se faire autour de l'idée de "projection » : cette notion, centrale dans le manifeste, peut renvoyer à la volonté d'arriver à une forme d'immédiateté, par-delà le langage articulé, via une utilisation du signe dans sa pure matérialité graphique ou sonore, d'une part, et, d'autre part, l'idée de l'œuvre finale, poème ou tableau, comme trace d'un événement. Pour Harold Rosenberg, dans l'expressionnisme abstrait, la toile «était devenue arène où agir - plutôt qu'espace où reproduire, reconstruire, analyser ou "exprimer" un objet, réel ou imaginaire. Ce qui devait passer sur la toile, ce n'était plus une image mais un fait, une action» (Rosenberg [1952] 1997 : 644).

Enregistrer sa propre activité physique, c'est, précisément, l'ambition que se donne à partir de 1953 François Dufrêne, poète et plasticien issu du lettrisme. Cette poésie, issue du phonétisme, se centre sur les "sons bruts", "sons pulsifs, émotions pures", 
imposant, selon Arnaud Labelle-Rojoux, "le parallèle» avec "l'action painting: Dufrêne est dans sa voix comme Pollock est dans sa peinture. Il a le cri-rythme dans la peau » (Labelle-Rojoux [1988] 2004 : 232). Dufrêne compare quant à lui ses crirythmes à la peinture gestuelle de Mathieu. Certaines tendances de la poésie visuelle vont dans un sens comparable, qui donnent à voir le geste d'écriture même. Pierre et Ilse Garnier publient en 1965 un ensemble de dactylopoèmes sous le titre Poèmes mécaniques. Le poème, composé de lettres isolées ou de mots diffractés dans l'espace de la page, se fait "événement", inscrivant sur la page "une aventure » tout en rythmes visuels, que Pierre Garnier rapporte à l'action painting de Jackson Pollock. Dès lors, l'œuvre se veut point d'ancrage provisoire d'énergies : «action writing». Ainsi, selon la formule de Pierre Garnier dans « Position 2 du Spatialisme », «le processus de création du poème est devenu le poème lui-même. Ce qui naguère était pris dans sa forme est aujourd'hui donné dans sa force» (Garnier [1964b] 1968: 144). Le poème est un ensemble de "gestes que nous inscrivons dans l'espace ». L'intégration du médium dans le processus de constitution du poème se lie à la recherche d'une forme d'immédiateté, qui envisage la bande magnétique ou la page comme surface de projection, et fait du poème un événement.

Cette conception n'est cependant pas intrinsèquement liée à l'abstraction, et le texte / manifeste dessine une orientation différente, qui marquerait le passage, chez Heidsieck d'une écriture-action (action writing) à une "poésie action", l'éloignant d'autant du projet spatialiste pour le rapprocher d'une autre sensibilité picturale, celle du Nouveau Réalisme. Le geste de prélèvement et déplacement des bruits opéré dans Couper n'est pas jouer, selon le modèle de la «biopsie », n'est en effet pas sans rappeler l'ambition sociologique des peintres du mouvement fondé par Pierre Restany et si Heidsieck ne fait pas partie du groupe, il en fréquente certains acteurs comme Dufrêne, Hains, Villéglé ou Niki de Saint-Phalle : le premier disque d'Heidsieck paraît dans un numéro de la revue KWY (1963) consacré aux Nouveaux Réalistes, et Couper n'est pas jouer est utilisé plus tard par Villéglé comme bande-son de son film Un mythe dans la ville (1974-2002).

Ainsi, « au-delà des dadaïsmes, surréalismes et tous autres isthmes» (Chopin [1966] 1993), les liens se créent et les réseaux de sensibilités se tissent sans former mouvement, ce que les modalités d'écriture et de diffusion des différents manifestes laissent clairement apparaître. À cet égard, les stratégies d'un Julien Blaine, lui aussi bâtisseur de réseaux via sa revue $\operatorname{DOC}(K) \mathcal{S}^{12}$ à partir de 1976, sont révélatrices lorsqu'il multiplie les épithètes pour désigner sa propre pratique: poésie "séméiotique ", "deux points", "élémentaire", puis "en chair et en os». Ces appellations, revendiquées dans autant de manifestes, témoignent d'une posture expérimentale, mue par une volonté de défigement, et un refus du groupe constitué au profit d'une logique souple de "réseau ", dont la communication non centralisée par mail art est la figure : "Nous participions certes d'une parole collective, mais toujours individuellement, sans jamais obéir aux règles d'un groupe " (Blaine 2009: 45). Mais ce que laisse surtout apparaître avec éclat l'examen des manifestes publiés par Blaine et Heidsieck, qui les distingue d'une manière plus fondamentale des stratégies de Pierre Garnier, est l'expression et la mise en œuvre d'un faire spécifique à la poésie action. 


\section{Agir la poésie} performances datant de 1962. Mais en 1961, pour ce qui restera l'unique diffusion publique du poème « D3Z », lors du vernissage de l'exposition 7 Métasignes sur la fleur de Jean Degottex, un dispositif est mis en place qui trouvera par la suite une évolution dans la poésie performée. La diffusion associe en effet une composante visuelle à la composante sonore, celle-là même que le poète recherche dans ses performances :

Ce que je cherche toujours, c'est d'offrir la possibilité à l'auditeur/spectateur de trouver un point de focalisation et de fixation visuelle. Cela me paraît essentiel. Sans aller jusqu'au happening loin de là, je propose toujours un minimum d'action pour que le texte se présente comme une chose vivante et immédiate et prenne une texture quasiment physique. Il ne s'agit donc pas de lecture à proprement parler, mais de donner à voir le texte entendu. (Heidsieck 1976 : 131)

Une convergence se produit entre une gestualité dont les tableaux portent indice, une gestualité sonore, et un ancrage visuel, dont la perception est en outre remodelée par la lecture du manifeste: comment ne pas voir, dans l'impressionnante verticalité des toiles grand format sur lesquelles sont tracés les métasignes, une incarnation de cette " poésie debout ", «dressée », sortie de l'horizontalité du livre, à laquelle se substituera celle du corps du poète ? Cette verticalité ne fait-elle pas écho au format de la grande feuille de papier sur laquelle s'imprime le texte / manifeste ? Degottex le souligne luimême dans un texte consacré aux métasignes: "J'ai toujours remarqué que la sensation d'être dans un tableau est surtout déterminée par une démesure dans le sens de la hauteur, par un dépassement dans le sens vertical [...] (Degottex $1992:$ 80). Des résonances s'établissent ainsi entre l'œuvre introduite par le manifeste, le texte de ce dernier, son support et ses conditions de diffusion, selon un fonctionnement qui joue à plein de la dimension performative de tout manifeste, mais déborde la seule relation programme / exemple de mise en application du programme, encore à l'œuvre dans les manifestes spatialistes. Le manifeste est «à la fois un programme et sa mise en œuvre » (Abastado $1980: 5$ ).

CEuvre à part entière, Couper n'est pas jouer fonctionne précisément de cette manière, non seulement parce que, en tant que poème sonore diffusé sur disque, il constitue une mise en application de la volonté d'«arracher le poème à la page » énoncée dans le manifeste, mais aussi dans la mesure où son écriture relève d'une mise en action. Le titre même, emprunté au vocabulaire du jeu de belote ou de dames («soufflé n'est pas joué »), littéralise l'expression pour décrire les actions mises en œuvre dans et par l'écriture, qui relève du cut-up sonore. La coupe est en effet au travail dans toute la pièce à plusieurs niveaux. Comme celui du premier manifeste «... pour un poème donc...", le texte commence comme une suite: «... bon, bon, BON... et puis... comment ? .... Et puis » : points de suspension et connecteur logique présupposent un début de phrase situé hors texte, une prise de parole antérieure dont il ne capterait qu'une suite, établissant de la sorte une absence de solution de continuité entre le texte et son «dehors ». Loin de se déployer en un discours continu et ordonné, le propos théorique est à son tour marqué par une forte oralité, avec de nombreuses ruptures de rythme, et présente la syntaxe déconstruite d'une parole en train de s'élaborer. Chaque ensemble commence par des points de suspension, comme une reprise de souffle, les onomatopées, les répétitions, les coupes, apocopes, reprises de termes, énumérations paratactiques, abondent : 
dialogue / clac-flash / clac / oui / surgi / clac oui / clac / surgi surgi né / au cœur / au cœur même / cœur absolu / fugace / fugace stop / [...] l'Absolu est bien davantage me semble-t-il - mille pardons / dans le souffle coupé d'un souffle coupé / ah / et son évidence même immédiate [...] ... souffle court / choc / coupé / coupé / court / choc / coupé / cloué / cloué / coupé / cloué

alternant avec des interruptions brutales, hésitations, retours, interjections : perfectibilité dans le conditionnement / eh! eh! / surtout n'y va.../ droits et devoirs/ Hep là... / où allez vous ? [...] Alors que... Alors que... tout cela est bien confus... Je tourne en rond. Suffit. En rond. Chaque mot me pousse vers son opposé. Chaque phrase vers... en rond. Vers... Il y a l'accident qui bascule toutes les prévisions. (Heidsieck [1968] 2005 : 18-19)

40 Le caractère événementiel de la prise de parole l'éloigne du discours théorique pour l'approcher d'une parole performative. Les longues énumérations de verbes à l'infinitif qui le ponctuent, déclinant autant d'actions que la poésie se doit d'entreprendre ${ }^{13}$ ne font donc pas que dessiner un programme, mais en constituent la mise en œuvre dans le temps même du manifeste.

Cette parole coupée, sectionnée, se confronte aux bruits du monde dans lequel le poète enjoint à faire sa « rentrée ». En effet le texte, mis en voix, est mixé à d'autres voix et d'autres bruits, enregistrés à même le réel social, plongé dans un bain sonore avec lequel il entre en confrontation via le montage. Couper n'est pas jouer fait partie de la série des «Biopsies", poèmes dont le principe est d'opérer des prélèvements dans le « corps social », via le magnétophone. Le premier mouvement de cette «triface » fait ainsi entendre, en alternance avec la voix du poète, des extraits d'enregistrement du vote de la motion de censure en mai 1968. Les fragments de discours de Pompidou, Chaban-Delmas, Giscard, etc. durant les débats, ainsi que les applaudissements et huées de l'assemblée, et la liste des députés énoncée par ordre alphabétique, sont audibles. Les coupes se font plus rapides dans le deuxième mouvement, où la voix du poète en écho sur les deux pistes est interrompue de sons divers, extraits d'un freejazz sauvage, bruits de chantiers, meuglements, grognements, cris de hyène, alternent avec des annonces promotionnelles et l'énoncé de gros titres de journaux («Un engagement dans la plaine des Joncs fait 161 morts ", " Une sonde soviétique se pose en douceur sur le sol de Vénus le 19 octobre $1967 »)$, pendant que la voix du poète énonce sa volonté de « couper court ». L'accumulation rapide des bruits finit par créer un effet de désordre, de vitesse incontrôlée, avant de s'arrêter brutalement pour laisser place à la voix seule, énonçant: "le poème face à cela à tout cela au monde». Enfin, dans le troisième mouvement, la "révolution permanente " appelée est scandée par la rumeur de la manifestation : «mai 1968 : les étudiants en marche slogans » annonce la partition, de plus en plus fort, accompagnés bientôt des commentaires d'un radio-reporter décrivant les événements en direct, au cœur de l'action violente avec une émotion qui confine à la « PANIQUE ». La parole poétique s'ancre ainsi dans le bain sonore de la société, s'y confond et s'y confronte, tant par ce qui s'y énonce que par ce qui s'y produit, et par son biais de diffusion, à l'image du récit enchâssé au sein du manifeste ${ }^{14}$, l'histoire de Jens Hansen qui, en charge :

[...] d'un puissant poste émetteur, voulut secouer sa propre torpeur et celle des États-Majors... Il se mit à transmettre en code des extraits du livre qu'il avait en poche : Tropique du Cancer d'Henry Miller... [...] Selon les termes du SHAPE ceci est un des exemples les plus caractéristiques du mauvais usage que l'on peut faire d'un poste émetteur militaire. (Heidsieck [1968] 2005 : 12-13) 
Dans ce récit se reflète la velléité de la poésie sonore d'agir à la manière d'un virus, s'emparant des moyens de communication pour faire sa rentrée dans le monde et y agir.

Au poème, en définitive, de se prouver lui-même. Qu'il fasse éclater son évidence et sa nécessité. Peu importe comment. Quitte même à ce que ce soit - à la limite - pour ne témoigner que de son inanité. [...] ... pourquoi chercher à cerner, assiéger, percer, saisir ce qui par essence, vous filera toujours entre les doigts... dans le sectionnement de la bande enregistrée. (Heidsieck [1968] 2005 : 20-21).

Le manifeste advient ainsi par le faire davantage que par le dire. Se réalise ici pleinement ce que décrit Christophe Kihm (2013 : 13) à propos des manifestes : "Cette association de l'art à la vie est immédiatement inscrite dans la parole-action, emblématique du manifeste, qui consacre une première fois cette union dans et par le poème.»

Cette parole action est pleinement à l'œuvre dans les manifestes postérieurs de Julien Blaine, où le programme esthétique cède entièrement la place à un appel à l'action, tourné vers les créateurs mais aussi le collectif à venir qu'est la " foule des anonymes ", amenés à tous devenir créateurs. Le manifeste pour la "poésie deux points " annonçait ainsi une poésie action, parce que sa visée est de faire réagir :

Deux points, cela veut dire que le véritable objet de la poésie, c'est ce qui se passe après qu'elle $a$ eu lieu, sa manifestation n'étant en soi qu'une étincelle, un épisode, un signal soluble. [...] Notre action n'est rien sans les réactions qu'elle provoque. (Blaine 2009 : 52)

Le tract "Plateforme de base pour les éveilleurs de jour et de nuit de la poésie ":" » précise que les « éveilleurs » s'engagent « à transformer le milieu dans lequel on baigne [...] - à détourner de leur but initial les moyens d'oppression et de communication au service du pouvoir pour les utiliser contre ce pouvoir [...] à parasiter le décor et le déroulement de la vie des gens pour les rendre conscients des réalités qui les entourent ", et, ce faisant, à inciter " les gens » à pratiquer eux-mêmes ces actions de parasitage, "cet éveil dans une perspective révolutionnaire ». Le manifeste ouvrant la rubrique du "Vrai Art Nouveau », tenue par Julien Blaine sous le nom de Jules Van dans les pages de Libération en 1975, est une apologie de la malversation et du détournement comme témoignages du génie créatif à l'œuvre au quotidien :

Du cubisme à l'art conceptuel, l'histoire de l'art de ce siècle est aussi chiante qu'une table de multiplication parce que l'invention et le génie créatif ne sont plus du côté de ces «écoles» [...] L'invention c'est les mille et une façons d'exercer la malversation, les mille et un trucs pour voler le patron, les mille et une manières de se marrer à ses dépens. (Cité dans Blaine 2009 : 73)

En 1978, le «Manifeste pour l'occupation des stèles et socles abandonnés », publié sous forme d'affiche et envoyé dans le réseau mail art, procède d'une même logique, assorti cette fois d'un appel à l'action centré sur un objet particulier du décor urbain :

Pour donner une leçon à ceux qui ont oublié les gloires anciennes pour créer des gloires nouvelles; je réclame l'occupation des stèles et socles abandonnés. Présentez-vous sur le socle de votre choix, nu ou habillé et mettez-vous en valeur avec ou sans outils, vos instruments, vos jeux ou vos montures. (Cité dans Blaine $2009: 148)$

Recueillis dans les pages du quotidien, celles de la revue DOCKS (entre 1979 et 1984) ou dans la collection «les Anartistes" des Nouvelles Éditions Polaires dédiée "à une poésie de l'action » comme Processus de déculturatisation, les témoignages de réalisations 
suscitées par le manifeste forment alors un réseau informel, anonyme, qui sont pour le poète « les faire constitutifs d'une poésie considérée comme un art total », inséparable de l'activisme politique. Le travail de médiatisation est ainsi «destiné à donner visibilité et sens à un événement construit de toutes pièces collectivement " (Hanna [2006] $2015:$ 189).

Arpenter le domaine des poésies expérimentales au cours des années 1960-1970 par le prisme du manifeste autorise ainsi la mise en évidence d'une évolution dans les stratégies de mise en visibilité et d'occupation du champ. Si le programme d'une sortie du livre semble réunir ces pratiques, le modèle avant-gardiste du mouvement artistique est tenu à distance au profit d'une logique de réseau: la parole manifestaire est individuelle et polyphonique, et vise non pas à s'exprimer au nom d'un groupe, mais à agir et susciter l'action. En ce sens, le passage d'un modèle historique, représenté par la tentative spatialiste, aux manifestes performés qui sont action autant qu'appels à l'action, témoigne d'un projet maintenu de fusion de l'art et de la vie caractéristique des avant-gardes, et laisse apparaître la dimension éminemment politique de la poésie action. "Alors s'infiltrer dans les rouages et poulies de nos rythmes, sociaux et personnels. Panique. Se coller à leurs pulsations. Et puis... » (Heidsieck [1968] 2005 : 42).

\section{BIBLIOGRAPHIE}

Abastado, Claude, 1980, «Introduction à l'analyse des manifestes », Littérature, vol. 39, nº 3, p. 3-11.

Blaine, Julien, Schifres, Alain et Moineau, Jean-Claude, 1971, « La poésie hors du livre hors du spectacle hors de l'objet », Robho, $\mathrm{n}^{\circ}$ 5-6.

Blaine, Julien, 2009, Blaine au MAC : un tri, Limoges, Al Dante.

Bürger, Peter, [1974] 2013, Théorie de l'avant-garde, trad. Jean-Pierre Cometti, Paris, Questions théoriques, coll. « Saggio Casino ».

Cariou, André (dir.), 2008, Jean Degottex [Musée des Beaux-arts, Quimper, 4 juillet-30 septembre 2008 , etc.], Lyon, Fage.

Chopin, Henri, 1963, « Les mutations poétiques », Les Lettres, n 30, $2^{\mathrm{e}}$ trimestre, p. 11-14.

Chopin, Henri, [1966] 1993, « Au-delà des dadaïsmes, surréalismes et tous autres isthmes par des forces d'amour ", dans Poésure et peintrie, d'un art l'autre, Réunion des Musées nationaux/Musées de Marseille, p. 548.

Degottex, Jean, 1992, Degottex. Signes et métasignes 1954-1967, Nîmes, Carré d'art, Musée d'art contemporain de Nîmes.

Donguy, Jacques, 1993, « Entretien avec Pierre Garnier », Poésure et peintrie, d'un art l'autre, Réunion des Musées nationaux, Musées de Marseille, p. 390-397. 
Garnier, Pierre, 1963a, « Manifeste pour une poésie nouvelle, visuelle et phonique », Les Lettres, $\mathrm{n}^{\circ}$ $29,1^{\text {er }}$ trimestre, p. 1-8.

Garnier, Pierre, 1963b, « Deuxième manifeste pour une poésie visuelle », Les Lettres, $n^{\circ} 30,2^{\mathrm{e}}$ trimestre, p. 14-23.

Garnier, Pierre, 1963c, « Note liminaire - Plan pilote fondant le Spatialisme », Les Lettres, $\mathrm{n}^{\circ} 31,4^{\mathrm{e}}$ trimestre, p. 1-5.

Garnier, Pierre, 1964a, «Position 1 du Mouvement international », Les Lettres, $\mathrm{n}^{\circ} 32,2^{\mathrm{e}}$ trimestre, p. 1-5.

Garnier, Pierre, [1964b] 1968, « Position 2 du Spatialisme », Les Lettres, $n^{\circ} 33,4^{\mathrm{e}}$ trimestre, p. 1-2.

Garnier, Pierre, 1968, Spatialisme et poésie concrète, Paris, Gallimard.

Gleize, Jean-Marie, 1980, « Manifestes, préfaces. Sur quelques aspects du prescriptif », Littérature, vol. $39, \mathrm{n}^{\circ} 3, \mathrm{p} .12-16$.

Hanna, Christophe, [2006] 2015, «À propos de l'occupation des stèles et des socles abandonnés ", dans G. Suzanne (dir.), La Poésie à outrance. À propos de la poésie élémentaire de Julien Blaine, Dijon, Les Presses du réel, coll. « L'écart absolu chantiers », p. 189-191.

Heidsieck, Bernard, 1962, "Pour un poème debout... ", Cinquième saison, n 6, (1973, D2+D3Z, poèmes partitions de B.H (sur des peintures de Jean Degottex, Henri Chopin, coll. « Ou »;1980, $\operatorname{DOC}(K) S, \mathrm{n}^{\circ} 27 ; 1981$, Precisely $\mathrm{n}^{\circ}$ 10/11/12 (USA) ; 1998, DOC(K)S $\mathrm{n}^{\circ}$ 17/18/19/20, nouvelle série.).

Heidsieck, Bernard, 2001, Notes Convergentes, Romainville, Al Dante, coll. «\&».

Heidsieck, Bernard, [1968] 2005, Couper n'est pas jouer, Romainville, Al Dante.

Heidsieck, Bernard, 2008, «Introduction », dans A. Cariou (dir.), Jean Degottex [Musée des Beauxarts, Quimper, 4 juillet-30 septembre 2008, etc.], Lyon, Fage.

Heidsieck, Bernard, 2009, « Lettre à Jean Fournier d'octobre 1958, directeur de la galerie Kléber, Paris, où exposait à cette date Jean Degottex », Poèmes-partitions (1955-1965) précédé de Sitôt dit (1955), Romainville, Al Dante, p. 271-272.

Heidsieck, Bernard, Lemaire, Gérard-Georges et Mikiriammos, Philippe, 1976, « Quatrième entretien », dans W. S. Burroughs et B. Gysin, Colloque de Tanger, Paris, Christian Bourgois, p. 361.

Kihm, Christophe, 2013, « Manifestes, collectifs, mobilisations », Lignes, n 40, p. 9-20.

Labelle-Rojoux, Arnaud, [1988] 2004, L'Acte pour l'art, Romainville, Al Dante, coll. « \&».

Margel, Serge, 2013, « Le temps du manifeste », Lignes, $\mathrm{n}^{\circ}$ 40, p. 5-7.

Rosenberg, Harold, [1952] 1997, « Les peintres d'action américains », dans C. Harrison et P. Wood (dir.), Art en théorie 1900-1990, Paris, Hazan, p. 643-647.

Royère, Anne-Christine, 2018, « Trois poètes dans le laboratoire des poésies nouvelles : Ilse Garnier, Pierre Garnier et Henri Chopin ", Actes du colloque international Pierre et Ilse Garnier, deux poètes face au monde, 6-7 octobre 2016, Tours, Université François-Rabelais. À paraître aux Presses universitaire François Rabelais.

Tomiche, Anne, 2005, " "Manifestes" et "avant-gardes" au Xx $x^{\mathrm{e}}$ siècle ", Manières de critiquer ; colloque sur les avant-gardes à l'Université d'Artois, [En ligne], <halshs-00112236>.

Troin-Guis, Anysia, 2014, « Politique et poétique des revues », dans G. Suzanne (dir.), La Poésie à outrance. À propos de la poésie élémentaire de Julien Blaine, Dijon, Les Presses du réel, coll. «L'écart absolu chantiers ", p. 105-122. 
Vouilloux, Bernard, 2004, « Manifester la peinture. André Breton dans l'image », dans Tableaux d'auteurs : après l'ut pictura poesis, Saint-Denis, Presses Universitaires de Vincennes, p. 124-145.

\section{NOTES}

1. Pierre Garnier devient rédacteur en chef de la revue Les Lettres à partir de 1962.

2. Julien Blaine a fondé et dirigé de nombreuses revues à partir de 1962 dont Les Carnets de l'Octéor, Approches, Robho, et l'incontournable $D O C(K) S$ à partir de 1976. L'on peut également citer Jean-François Bory, co-directeur de la revue Approches avec Blaine en 1966, puis d'Agentzia et à partir de 1971 de L'Humidité.

3. "Manifeste pour une poésie nouvelle, visuelle et phonique ", Les Lettres, $n^{\circ} 29,1^{\mathrm{er}}$ trimestre 1963 ; «Deuxième manifeste pour une poésie visuelle », Les Lettres, $\mathrm{n}^{\circ} 30,2^{\mathrm{e}}$ trimestre 1963 , p. 14-23; « Note liminaire - Plan pilote fondant le Spatialisme », Les Lettres, $\mathrm{n}^{\circ} 31,4^{\mathrm{e}}$ trimestre 1963, p. 1-5; «Position 1 du Mouvement international », Les Lettres, $\mathrm{n}^{\circ} 32,2^{\mathrm{e}}$ trimestre 1964, p. 1-5; « Position 2 du Spatialisme », Les Lettres, no 33, $4^{\text {e }}$ trimestre 1964, p. 1-2.

4. A paru dans les livres : D2+D3Z, poèmes partitions de B.H (sur des peintures de Jean Degottex, Henri Chopin, coll. «Ou », 1973); et revues : Cinquième saison, ${ }^{\circ}$ 6, 1962 ; DOC (K)S, $\left.\mathrm{n}^{\circ} 27,1980\right)$; Precisely, n 10/11/12 (USA) 1981; DOC(K)S, n 17/18/19/20, nouvelle série, 1998.

5. Le sous-titre est en effet "C'est une préface ». Première parution: Trois biopsies + un passepartout, LP, 1971.

6. La distinction est de fait fragile: « les circonstances historiques et la réception des textes, la manière dont ils sont entendus, lus, interprétés, entraînent des glissements de qualifications : la préface de Poisson soluble devient vite le premier manifeste du Surréalisme », souligne Claude Abastado (1980: 5).

7. Les manifestes ont été à nouveau publiés en 1968 dans Spatialisme et poésie concrète: les numéros de page renvoient à cette édition postérieure.

8. Publié intégralement dans le recueil Notes Convergentes en 2001.

9. Pour une étude complète des relations entre Pierre Garnier et Henri Chopin au cours de cette période et après, voir Anne-Christine Royère, «Trois poètes dans le laboratoire des poésies nouvelles : Ilse Garnier, Pierre Garnier et Henri Chopin », actes du colloque international Pierre et Ilse Garnier, deux poètes face au monde, 6-7 octobre 2016, Tours, Université François-Rabelais. À paraître aux Presses Universitaire François Rabelais en 2018.

10. Les co-signataires sont: Mario Chamie, Carlfriedrich Claus, Ian Hamilton Finlay, Fujitomi Yasuo, John Furnival, Ilse Garnier, Pierre Garnier, Eugen Gomringer, Bohumila Grögerova, Josef Hirsal, Anselm Hollo, Sylveste Houédard, Ernst Jandl, Kitasono Katue, Frans van der Linde, E.M de Melo e Castro, Franz Mon, Edwin Morgan, Ladislav Novak, Herbert Read, Toshihik Schimizu, L.C. Vinholes, Paul de Vree, Emmett Williams, Jonathan Williams.

11. Le développement suivant est issu d'une étude consacrée aux relations de Bernard Heidsieck et Jean Degottex : Gaëlle Théval, « Frapper les mêmes touches de l'être », communication dans le cadre du colloque « Le poème d'art » (org. Serge Linarès), Université de Versailles Saint-Quentinen-Yvelines, 2-3 juin 2016.

12. Sur Julien Blaine revuiste, voir Anysia Troin-Guis (2014).

13. " et des gradins, ces gradins, toujours, pour descendre, s'agripper, descendre, et s'infiltrer, s'insinuer, ou plonger et descendre, descendre ou grimper, avancer, plus avant, plus avant, plus profondément, s'accrocher, se glisser, grimper, progresser, vaille que vaille, ces marches, oui... » 14. ... qui fait aussi l'objet du poème-partition « Chapeau ! ». 


\section{RÉSUMÉS}

Notre étude se focalise sur trois manifestes, qui apparaîtront comme des balises pour arpenter le champ et l'histoire des poésies expérimentales en France, produits entre 1961 et 1978 par trois figures centrales du domaine, Pierre Garnier, Bernard Heidsieck et Julien Blaine. C'est tant aux contenus critiques et programmatiques qu'aux modalités d'action de ces manifestes que nous nous intéressons, s'il est entendu que le manifeste ne saurait se réduire à un genre mais est « un geste, un acte", relevant "prioritairement d'une pragmatique du discours, d'une lecture sociologique, d'une analyse en termes de stratégies d'intervention dans le champ de l'institution » (Gleize $1980: 12$ ). Qu'en est-il de l'acte manifestaire dans la poésie expérimentale et en particulier dans la «poésie action"? Notre propos s'articule autour de trois actions, trois «faire » dont il s'agit d'observer les modalités : sortir du livre, où les programmes énoncés nous arrêtent, faire exister un groupe, où les modes de circulation et de réception ainsi que les impacts de ces manifestes sont envisagés, et agir la poésie, où la spécificité du mode d'agir des manifestes de poésie action est interrogée.

Our study focuses on three manifestos, produced between 1961 and 1978 by three central figures in the field, Pierre Garnier, Bernard Heidsieck and Julien Blaine, which appear as milestones for mapping the field and history of experimental poetry in France. We are interested to the critical and programmatic contents as much as to the modes of action of these manifestos: indeed, the manifesto can not be reduced to a literary genre but is "a gesture, an act" "primarily a pragmatics of discourse, a sociological reading, an analysis in terms of intervention strategies in the field of the institution" (Gleize 1980: 12). What about the act of demonstration in experimental poetry and especially in "action poetry"? Our purpose is articulated around three actions: to quit the book, to make a group exist, and to put poetry in action.

\section{INDEX}

Keywords : manifesto, spatialism, sound poetry, action poetry, experimental poetry, performance, avant-garde

Mots-clés : manifeste, spatialisme, poésie sonore, poésie action, poésie expérimentale, performance, avant-garde

\section{AUTEUR}

GAËLLE THÉVAL

Université Sorbonne Nouvelle - Paris 3, Thalim (UMR 7172) 